\title{
POSITIVE DEFINITE FUNCTIONS AND SHARP INEQUALITIES FOR PERIODIC FUNCTIONS
}

\author{
Viktor P. Zastavnyi \\ Donetsk National University, Donetsk \\ zastavn@rambler.ru
}

\begin{abstract}
Let $\varphi$ be a positive definite and continuous function on $\mathbb{R}$, and let $\mu$ be the corresponding Bochner measure. For fixed $\varepsilon, \tau \in \mathbb{R}, \varepsilon \neq 0$, we consider a linear operator $A_{\varepsilon, \tau}$ generated by the function $\varphi$ :

$$
A_{\varepsilon, \tau}(f)(t):=\int_{\mathbb{R}} e^{-i u \tau} f(t+\varepsilon u) d \mu(u), \quad t \in \mathbb{R}, \quad f \in C(\mathbb{T}) .
$$

Let $J$ be a convex and nondecreasing function on $[0,+\infty)$. In this paper, we prove the inequalities

$$
\left\|A_{\varepsilon, \tau}(f)\right\|_{p} \leqslant \varphi(0)\|f\|_{p}, \quad \int_{\mathbb{T}} J\left(\left|A_{\varepsilon, \tau}(f)(t)\right|\right) d t \leq \int_{\mathbb{T}} J(\varphi(0)|f(t)|) d t
$$

for $p \in[1, \infty]$ and $f \in C(\mathbb{T})$ and obtain criteria of extremal function. We study in more detail the case in which $\varepsilon=1 / n, n \in \mathbb{N}, \tau=1$, and $\varphi(x) \equiv e^{i \beta x} \psi(x)$, where $\beta \in \mathbb{R}$ and the function $\psi$ is 2-periodic and positive definite. In turn, we consider in more detail the case where the 2-periodic function $\psi$ is constructed by means of a finite positive definite function $g$. As a particular case, we obtain the Bernstein-Szegö inequality for the derivative in the Weyl-Nagy sense of trigonometric polynomials. In one of our results, we consider the case of the family of functions $g_{1 / n, h}(x):=h g(x)+(1-1 / n-h) g(n x)$, where $n \in \mathbb{N}, n \geq 2,-1 / n \leq h \leq 1-1 / n$, and the function $g \in C(\mathbb{R})$ is even, nonnegative, decreasing, and convex on $(0,+\infty)$ with supp $g \subset[-1,1]$. This case is related to the positive definiteness of piecewise linear functions. We also obtain some general interpolation formulas for periodic functions and trigonometric polynomials which include the known interpolation formulas of M. Riesz, of G. Szegö, and of A.I. Kozko for trigonometric polynomials.
\end{abstract}

Key words: Positive definite function, Trigonometric polynomial, Weyl-Nagy derivative, Bernstein-Szegő inequality, Interpolation formula.

\section{Introduction}

The role of positive definite functions in obtaining sharp inequalities for trigonometric polynomials and entire functions is well known (see, for instance, Boas [6, Ch. 11], Timan [22, Sect. 4.8], Lizorkin [13], Gorin [9], and Trigub and Belinsky [23]). For instance, the classical Bernstein inequality $\max \left|f^{\prime}(x)\right| \leq n \max |f(x)|$ for trigonometric polynomials of degree at most $n$ is related to the positive definiteness of the function $(1-|x|)_{+}$. A historical survey of such inequalities and the methods of their proof are given in the works by Gorin [9], Arestov and Glazyrina [5], Gashkov [8], and Vinogradov [25]. In the present paper, we obtain sharp inequalities for continuous periodic functions and, in particular, for trigonometric polynomials. These inequalities are related to positive definite functions. As consequences, we obtain generalizations of Bernstein-Szegö inequalities. We give criteria and descriptions of extremal functions in these inequalities.

A complex-valued function $f: \mathbb{R} \rightarrow \mathbb{C}$ is called positive definite on $\mathbb{R}(f \in \Phi(\mathbb{R}))$ if, for any $m \in \mathbb{N}$, any set of points $\left\{x_{k}\right\}_{k=1}^{m} \subset \mathbb{R}$, and any complex numbers $\left\{c_{k}\right\}_{k=1}^{m} \subset \mathbb{C}$, the following inequality holds:

$$
\sum_{k, j=1}^{m} c_{k} \overline{c_{j}} f\left(x_{k}-x_{j}\right) \geqslant 0 .
$$

It is easy to verify that, for any $\beta \in \mathbb{R}$, the function $f(x)=e^{i \beta x}$ is positive definite. For a function in $\Phi(\mathbb{R})$, the continuity at zero is equivalent to the continuity on $\mathbb{R}$. If $f, g \in \Phi(\mathbb{R})$, then $|f(x)| \leq f(0)$, 
$\overline{f(-x)}=f(x),|f(x+y)-f(x)|^{2} \leq 2 f(0)(f(0)-\operatorname{Re} f(y)), x, y \in \mathbb{R}$, and $\bar{f}, \operatorname{Re} f, f g \in \Phi(\mathbb{R})$. In 1932, S. Bochner and, independently, A. Khinchin proved the following criterion of positive definiteness.

Theorem 1 (Bochner-Khinchin). The inclusion $f \in \Phi(\mathbb{R}) \cap C(\mathbb{R})$ holds if and only if there exists a finite nonnegative Borel measure $\mu$ on $\mathbb{R}$ such that

$$
f(x)=\int_{\mathbb{R}} e^{i x t} d \mu(t), \quad x \in \mathbb{R} .
$$

The proof of this theorem can be found, for instance, in $[2,7,19,23,24]$. As a direct consequence, we obtain the following criterion of positive definiteness in terms of nonnegativity of the Fourier transform: if $f \in C(\mathbb{R}) \cap L_{1}(\mathbb{R})$, then $f \in \Phi(\mathbb{R}) \Longleftrightarrow \widehat{f}(t) \geq 0, t \in \mathbb{R}$, where

$$
\widehat{f}(t):=\int_{\mathbb{R}} e^{-i t x} f(x) d x, \quad t \in \mathbb{R} .
$$

Using this criterion, it is not difficult to see that the functions $(1-|x|)_{+}, e^{-|x|}$, and $e^{-x^{2}}$ are positive definite.

We denote by $C(\mathbb{T}), \mathbb{T}:=[-\pi, \pi]$, the class of $2 \pi$-periodic continuous functions $f: \mathbb{R} \rightarrow \mathbb{C}$. For $f \in C(\mathbb{T})$, we define

$$
\|f\|_{\infty}:=\sup \{|f(t)|: t \in \mathbb{T}\} \text { and }\|f\|_{p}:=\left(\int_{\mathbb{T}}|f(t)|^{p} d t\right)^{1 / p}, \quad 1 \leq p<\infty .
$$

Let $\varphi \in \Phi(\mathbb{R}) \cap C(\mathbb{R})$, and let $\mu$ be the corresponding finite nonnegative Borel measure on $\mathbb{R}$ such that

$$
\varphi(x)=\int_{\mathbb{R}} e^{i x u} d \mu(u), \quad x \in \mathbb{R} .
$$

For fixed $\varepsilon, \tau \in \mathbb{R}, \varepsilon \neq 0$, we consider the linear operator $A_{\varepsilon, \tau}$ generated by the function $\varphi$ :

$$
A_{\varepsilon, \tau}(f)(t):=\int_{\mathbb{R}} e^{-i u \tau} f(t+\varepsilon u) d \mu(u), \quad t \in \mathbb{R}, \quad f \in C(\mathbb{T}) .
$$

The function $A_{\varepsilon, \tau}(f)(t)$ is continuous on $\mathbb{R}$ and $2 \pi$-periodic. Therefore, $A_{\varepsilon, \tau}: C(\mathbb{T}) \rightarrow C(\mathbb{T})$. In this paper, we prove the inequalities

$$
\left\|A_{\varepsilon, \tau}(f)\right\|_{p} \leqslant \varphi(0)\|f\|_{p}, \quad \int_{\mathbb{T}} J\left(\left|A_{\varepsilon, \tau}(f)(t)\right|\right) d t \leq \int_{\mathbb{T}} J(\varphi(0)|f(t)|) d t,
$$

where $1 \leqslant p \leqslant \infty, f \in C(\mathbb{T})$, and $J$ is a convex nondecreasing function on $[0,+\infty)$. In addition, we obtain some criteria of extremal function in these inequalities (see Theorems 2 and 4 and Remark 2). We study in more detail the case in which $\varepsilon=1 / n, n \in \mathbb{N}, \tau=1$, and $\varphi(x) \equiv e^{i \beta x} \psi(x)$, where $\beta \in \mathbb{R}$ and $\psi$ is a 2-periodic function of the class $\Phi(\mathbb{R}) \cap C(\mathbb{R})$ (see Theorem 5 and Remarks 4 and 5 ). In turn, we consider in more detail the case where a 2-periodic function $\psi$ is constructed by means of a finite function $g \in \Phi(\mathbb{R}) \cap C(\mathbb{R})$ (Theorem 6). As a particular case, we obtain the Berstein-Szegö inequality for the Weyl-Nagy derivative of trigonometric polynomials (Remark 7). In Theorem 8, we consider the case of the family of functions $g_{1 / n, h}(x):=h g(x)+(1-1 / n-h) g(n x)$, where $n \in \mathbb{N}, n \geq 2,-1 / n \leq h \leq 1-1 / n$, and the function $g \in C(\mathbb{R})$ is even, nonnegative, decreasing, and convex on $(0,+\infty)$ with $\operatorname{supp} g \subset[-1,1]$. This case is related to the positive definiteness of piecewise linear functions [15]. In Theorem 9 and Corollary 3, we obtain general interpolation formulas for periodic functions which include the known interpolation formulas of M. Riesz, of G. Szegö, and of A.I. Kozko [11] for trigonometric polynomials (see Remark 8). 


\section{Auxiliary facts of measure and integration theory}

We recall some well-known facts which are used in the paper to describe extremal functions. In this section, a measure $\mu$ is a nonnegative countably additive function defined on a $\sigma$-algebra $\gamma$ with identity element $\Omega$. For $p \in(0,+\infty)$, the class $L_{p}(\Omega, \gamma, \mu)$ is the set of all $\gamma$-measurable functions $f: \Omega \rightarrow \mathbb{C}$ such that

$$
\|f\|_{p}:=\left(\int_{\Omega}|f(u)|^{p} d \mu(u)\right)^{1 / p}<+\infty .
$$

The class $L_{\infty}(\Omega, \gamma, \mu)$ is the set of all $\gamma$-measurable functions $f: \Omega \rightarrow \mathbb{C}$ for which there exists $K=K(f)<+\infty$ such that $|f(u)| \leq K$ for $\mu$-almost every $u \in \Omega$. For $f \in L_{\infty}(\Omega, \gamma, \mu)$, the norm is defined by the formula

$$
\|f\|_{\infty}:=\inf \{K:|f(u)| \leq K \text { for } \mu \text {-almost all } u \in \Omega\} .
$$

For convenience, we assume that $L_{p}(\Omega, \gamma, \mu)=L_{p}(\Omega, \mu)=L_{p}(\Omega)$.

Proposition 1. Let $(\Omega, \gamma, \mu)$ be a measurable space with measure. If $f \in L_{1}(\Omega, \mu)$, then

$$
\left|\int_{\Omega} f(u) d \mu(u)\right| \leq \int_{\Omega}|f(u)| d \mu(u)
$$

and the inequality turns into an equality if and only if the equality $f(u)=e^{i \theta}|f(u)|$ holds for some $\theta \in \mathbb{R}$ and for $\mu$-almost all $u \in \Omega$.

P r o o f. See, for instance, [18, Theorems 1.33 and 1.39]. Obviously, for some $\beta \in \mathbb{R}$, we have

$$
\left|\int_{\Omega} f(u) d \mu(u)\right|=e^{i \beta} \int_{\Omega} f(u) d \mu(u)=\int_{\Omega} e^{i \beta} f(u) d \mu(u)=\int_{\Omega} \operatorname{Re}\left(e^{i \beta} f(u)\right) d \mu(u) \leq \int_{\Omega}|f(u)| d \mu(u)
$$

and the inequality turns into an equality if and only if $\operatorname{Re}\left(e^{i \beta} f(u)\right)=|f(u)|$ for $\mu$-almost all $u \in \Omega$ or if and only if $e^{i \beta} f(u)=|f(u)|$ for $\mu$-almost all $u \in \Omega$.

Proposition 2. Assume that $J$ is a convex function on $\mathbb{R},(\Omega, \gamma, \mu)$ is a measurable space with finite measure, $\mu(\Omega)>0$, and $f$ is a real-valued function in $L_{1}(\Omega, \mu)$. Then

$$
J\left(\frac{1}{\mu(\Omega)} \int_{\Omega} f(u) d \mu(u)\right) \leq \frac{1}{\mu(\Omega)} \int_{\Omega} J(f(u)) d \mu(u) .
$$

If the function $J$ is strictly convex at the point $\alpha=\int_{\Omega} f(u) d \mu(u) / \mu(\Omega)$, then equality in (2.1) is attained if and only if $f(u)=\alpha$ for $\mu$-almost all $u \in \Omega$.

For a proof of this result, see, for instance, [12, Sect. 2.2].

The next proposition will be needed only in Remark 3 .

Proposition 3. Let $(\Omega, \gamma, \mu)$ be a measurable space with measure. Then:

(i) if, for some $q>0$, we have $f \in L_{p}(\Omega)$ for all $p \in[q,+\infty)$ and $\liminf _{p \rightarrow+\infty}\|f\|_{p}<+\infty$, then $f \in L_{\infty}(\Omega)$ and $\|f\|_{\infty} \leq \liminf _{p \rightarrow+\infty}\|f\|_{p}$

(ii) if, for some $q>0$, we have $f \in L_{\infty}(\Omega) \cap L_{q}(\Omega)$, then $f \in L_{p}(\Omega)$ for all $p \in[q,+\infty)$ and $\|f\|_{\infty}=\lim _{p \rightarrow+\infty}\|f\|_{p}$ 
P r o o f. (i) We take a sequence $\left\{p_{n}\right\}, n \in \mathbb{N}$, such that $p_{n}>0, p_{n} \rightarrow+\infty$, and $\|f\|_{p_{n}} \rightarrow$ $c:=\liminf _{p \rightarrow+\infty}\|f\|_{p} \geq 0$. For an arbitrary $\sigma>c$, we define $\varepsilon:=(\sigma-c) / 2>0$. Then there exists a number $n(\sigma)$ such that the inequality $\|f\|_{p_{n}} \leq c+\varepsilon=(\sigma+c) / 2<\sigma$ holds for all $n \geqslant n(\sigma)$. The Chebyshev inequality implies that

$$
\mu(\{x \in \Omega:|f(x)| \geq \sigma\}) \leq\left(\frac{\|f\|_{p_{n}}}{\sigma}\right)^{p_{n}} \rightarrow 0, \quad n \rightarrow+\infty .
$$

Therefore, $|f(x)|<\sigma$ for $\mu$-almost all $x \in \Omega$ and, hence, $\|f\|_{\infty} \leq c$.

(ii) If $\|f\|_{q}=0$, the required assertion is obvious. Let $\|f\|_{q}>0$. Then, for any $p>q$, the inequality $\|f\|_{p} \leq\|f\|_{\infty}^{(p-q) / p}\|f\|_{q}^{q / p}$ holds. This inequality and assertion (i) yield

$$
\limsup _{p \rightarrow+\infty}\|f\|_{p} \leq\|f\|_{\infty} \leq \liminf _{p \rightarrow+\infty}\|f\|_{p} \leq \limsup _{p \rightarrow+\infty}\|f\|_{p} .
$$

\section{Sharp $L_{p}$-inequalities for periodic functions}

Equality (1.1) implies the inequality

$$
\left|A_{\varepsilon, \tau}(f)(t)\right| \leq \int_{\mathbb{R}}|f(t+\varepsilon u)| d \mu(u), \quad f \in C(\mathbb{T}), \quad t \in \mathbb{R} .
$$

Obviously, $\left\|A_{\varepsilon, \tau}(f)\right\|_{\infty} \leqslant \varphi(0)\|f\|_{\infty}$.

If $1 \leqslant p<\infty$, then inequality (3.1) along with the Minkowski inequality [12, Theorem 2.4] yields

$$
\begin{aligned}
\left\|A_{\varepsilon, \tau}(f)\right\|_{p}= & \left(\int_{\mathbb{T}}\left|A_{\varepsilon, \tau}(f)\right|^{p} d t\right)^{\frac{1}{p}} \leqslant\left(\int_{\mathbb{T}}\left(\int_{\mathbb{R}}|f(t+\varepsilon u)| d \mu(u)\right)^{p} d t\right)^{\frac{1}{p}} \\
& \leqslant \int_{\mathbb{R}}\left(\int_{\mathbb{T}}|f(t+\varepsilon u)|^{p} d t\right)^{\frac{1}{p}} d \mu(u)=\varphi(0)\|f\|_{p} .
\end{aligned}
$$

It follows from the Fubini theorem that the Fourier series of the function $A_{\varepsilon, \tau}(f)(t)$ has the form

$$
A_{\varepsilon, \tau}(f)(t) \sim \sum_{k \in \mathbb{Z}} \varphi(\varepsilon k-\tau) c_{k}(f) e^{i k t}, \quad f \in C(\mathbb{T}),
$$

where $c_{k}(f)$ are the Fourier coefficients of the function $f$ :

$$
c_{k}(f)=\frac{1}{2 \pi} \int_{-\pi}^{\pi} f(t) e^{-i k t} d t, \quad k \in \mathbb{Z} .
$$

Let us find sufficient conditions for the equality

$$
\left\|A_{\varepsilon, \tau}(f)\right\|_{p}=\varphi(0)\|f\|_{p} .
$$

If $|\varphi(\varepsilon s-\tau)|=\varphi(0)$ for some $s \in \mathbb{Z}$, then equality (3.3) holds for the polynomial $f(t)=c e^{i s t}$, $c \in \mathbb{C}$, since, in this case, $A_{\varepsilon, \tau}(f)(t)=\varphi(\varepsilon s-\tau) c e^{i s t}$. If $\tau / \varepsilon \in \mathbb{Z}$, this condition is satisfied for $s=\tau / \varepsilon$. 
If, for some $s, m \in \mathbb{Z}, s \neq m$, we have

$$
|\varphi(\varepsilon s-\tau)|=|\varphi(\varepsilon m-\tau)|=\varphi(0),
$$

then equality (3.3) holds for the polynomial $f(t)=c e^{i s t}+\nu e^{i m t}, c, \nu \in \mathbb{C}$, since, in this case,

$$
A_{\varepsilon, \tau}(f)(t)=\varphi(\varepsilon s-\tau) c e^{i s t}+\varphi(\varepsilon m-\tau) \nu e^{i m t} .
$$

We only need to take into account that, for any $\delta, \alpha \in \mathbb{R}$, the following equalities hold:

$$
\left\|c e^{i s t}+e^{i \delta} \nu e^{i m t}\right\|_{p}=\left\|c e^{i s(t+\alpha)}+e^{i \delta} \nu e^{i m(t+\alpha)}\right\|_{p}=\left\|c e^{i s t}+e^{i(\delta+m \alpha-s \alpha)} \nu e^{i m t}\right\|_{p} .
$$

In particular, the latter equality holds for $\alpha=\delta /(s-m)$.

If $\tau \neq 0,|\varphi(-2 \tau)|=\varphi(0), \varepsilon=\tau / n$, and $n \in \mathbb{N}$, then condition (3.4) is satisfied for $s=n$ and $m=-n$. Hence, $\left\|A_{\tau / n, \tau}(f)\right\|_{p}=\varphi(0)\|f\|_{p}$ for the polynomial $f(t)=c e^{i n t}+\nu e^{-i n t}$ with $c, \nu \in \mathbb{C}$.

Thus, we have proved the following theorem.

Theorem 2. Assume that $\varphi \in \Phi(\mathbb{R}) \cap C(\mathbb{R}), \tau, \varepsilon \in \mathbb{R}$, and $\varepsilon \neq 0$. Then:

1) the operator $A_{\varepsilon, \tau}$ acts from $C(\mathbb{T})$ to $C(\mathbb{T})$, is a multiplier, and satisfies the inequality

$$
\left\|A_{\varepsilon, \tau}(f)\right\|_{p} \leqslant \varphi(0)\|f\|_{p}, \quad 1 \leqslant p \leqslant \infty, \quad f \in C(\mathbb{T}) ;
$$

2) if, for some $s \in \mathbb{Z}$, the condition $|\varphi(\varepsilon s-\tau)|=\varphi(0)$ is satisfied, then equality in (3.5) is attained at the polynomials $f(t)=c e^{i s t}, c \in \mathbb{C}$. If $\tau / \varepsilon \in \mathbb{Z}$, this condition is satisfied for $s=\tau / \varepsilon$.

If, for some $s, m \in \mathbb{Z}, s \neq m$, condition (3.4) is satisfied, then equality in (3.5) is attained at the polynomials $f(t)=c e^{i s t}+\nu e^{i m t}, c, \nu \in \mathbb{C}$.

If $\tau \neq 0$ and $|\varphi(-2 \tau)|=\varphi(0)$, then equality in (3.5) for $\varepsilon=\tau / n, n \in \mathbb{N}$, is attained at the polynomials $f(t)=c e^{i n t}+\nu e^{-i n t}, c, \nu \in \mathbb{C}$.

Remark 1. In connection with the conditions in Theorem 2, the following simple property of positive definite functions is useful: if $\varphi \in \Phi(\mathbb{R})$ and, for some $y, \delta \in \mathbb{R}, y \neq 0$, we have $\varphi(y)=\varphi(0) e^{i \delta y}$, then $\varphi(x) \equiv f(x) e^{i \delta x}$, where $f \in \Phi(\mathbb{R})$ and $f$ is periodic with period $|y|>0$. Indeed, the function $f(x) \equiv \varphi(x) e^{-i \delta x}$ is the product of two positive definite functions. Therefore, $f \in \Phi(\mathbb{R})$ and, hence, for any $x \in \mathbb{R}$, we have

$$
|f(x+y)-f(x)|^{2} \leq 2 f(0)(f(0)-\operatorname{Re} f(y)) .
$$

Since $f(y)=\varphi(y) e^{-i \delta y}=\varphi(0)=f(0) \geq 0$, we have $f(x+y)-f(x)=0$ for all $x \in \mathbb{R}$. If, in addition, $\varphi \in C(\mathbb{R})$, then the Bochner measure of the function $\varphi$ is discrete and concentrated at the points $t_{k}=2 \pi k /|y|+\delta, k \in \mathbb{Z}$, and $\mu\left(\left\{t_{k}\right\}\right)=c_{k}(f) \geq 0, k \in \mathbb{Z}$ (see Theorem 3 below).

Remark 2. When $p=\infty$, inequality (3.5) turns into an equality at some function $f \in C(\mathbb{T})$ (see inequality (3.1) and Proposition 1) if and only if the equality $f(\xi+\varepsilon u)=e^{i(u \tau+\beta)}\|f\|_{\infty}$ holds for some $\xi, \beta \in \mathbb{R}$ and $\mu$-almost all $u \in \mathbb{R}$.

When $p=1$, inequality (3.5) turns into an equality at some function $f \in C(\mathbb{T})$ (see inequality (3.1) and Proposition 1) if and only if, for any $t \in \mathbb{R}$, there exists a number $\beta(t) \in \mathbb{R}$ such that the equality $f(t+\varepsilon u)=e^{i(u \tau+\beta(t))}|f(t+\varepsilon u)|$ holds for $\mu$-almost all $u \in \mathbb{R}$. This implies that if a function $f \in C(\mathbb{T})$ is extremal in inequality (3.5) with $p=1$, then any function of the form $c f(t) g(t)$, where $c \in \mathbb{C}, g \in C(\mathbb{T})$, and $g(t) \geq 0$ for all $t \in \mathbb{R}$, is also extremal.

When $p \in(1, \infty)$, inequality (3.5) turns into an equality at some function $f \in C(\mathbb{T})$ if and only if, for any $t \in \mathbb{R}$ and $\mu$-almost all $u \in \mathbb{R}$, the equality $f(t+\varepsilon u)=e^{i u \tau} c(t)$ holds, where 
$c(t)=A_{\varepsilon, \tau}(f)(t) / \varphi(0) \in C(\mathbb{T})\left(\right.$ for such $p$, see Theorem 4 below for $\left.J(t)=t^{p}\right)$.

Remark 3. If $1 \leq p<\infty$, the class $C(\mathbb{T})$ is everywhere dense in $L_{p}(\mathbb{T})$ (the Lebesgue measure is taken as a measure). Therefore, inequality (3.5) implies that the multiplier $A_{\varepsilon, \tau}: C(\mathbb{T}) \rightarrow C(\mathbb{T})$ defined by formula (3.2) is extended to the multiplier $A_{\varepsilon, \tau}: L_{p}(\mathbb{T}) \rightarrow L_{p}(\mathbb{T}), 1 \leq p<\infty$, and

$$
\left\|A_{\varepsilon, \tau}(f)\right\|_{p} \leqslant \varphi(0)\|f\|_{p}, \quad 1 \leqslant p<\infty, \quad f \in L_{p}(\mathbb{T}) .
$$

Hence, $A_{\varepsilon, \tau}: L_{\infty}(\mathbb{T}) \rightarrow L_{\infty}(\mathbb{T})$ and inequality (3.6) holds with $p=\infty$. We only need to use the well-known facts from measure and integration theory (see Proposition 3).

\section{Periodic positive definite functions}

The following description of periodic functions of the class $\Phi(\mathbb{R}) \cap C(\mathbb{R})$ is well known (see, for instance, [7, Theorem 1.7.5] and [10, Sect. II.1]).

Theorem 3. If $\psi \in C(\mathbb{R})$ and $\psi$ is $2 T$-periodic with $T>0$, then $\psi \in \Phi(\mathbb{R})$ if and only if $c_{k}(\psi) \geq 0, k \in \mathbb{Z}$, where

$$
c_{k}(\psi):=\frac{1}{2 T} \int_{-T}^{T} \psi(x) e^{-i \pi k x / T} d x, \quad k \in \mathbb{Z} .
$$

In this case, the function $\psi$ is expanded into the absolutely convergent Fourier series

$$
\psi(x)=\sum_{k \in \mathbb{Z}} c_{k}(\psi) e^{i \pi k x / T}, \quad x \in \mathbb{R} .
$$

Corollary 1. Assume that $f \in \Phi(\mathbb{R}) \cap C(\mathbb{R}), \operatorname{supp} f \subset[-1,1]$, and a 2-periodic function $\psi(x)$ coincides with the function $f(x)$ for $x \in[-1,1]$. Then $\psi \in \Phi(\mathbb{R}) \cap C(\mathbb{R})$ and $\psi(x-1)=$ $f(x-1)+f(x+1)$ for $x \in[-2,2]$.

P r o o f. Since $\psi( \pm 1)=f( \pm 1)=0$, we have $\psi \in C(\mathbb{R})$ and

$$
2 c_{k}(\psi)=\int_{-1}^{1} f(x) e^{-i \pi k x} d x=\widehat{f}(\pi k) \geq 0, \quad k \in \mathbb{Z} .
$$

Therefore, $\psi \in \Phi(\mathbb{R}) \cap C(\mathbb{R})$. Since supp $f \subset[-1,1]$, we obviously have

$$
\psi(x-1)=\sum_{k \in \mathbb{Z}} f(x-1+2 k), \quad x \in \mathbb{R} .
$$

Only terms with $k=0$ and $k=1$ remain in this sum for $x \in[-2,2]$.

\section{Sharp integral inequalities for periodic functions}

Let $\varphi \in \Phi(\mathbb{R}) \cap C(\mathbb{R})$ and $\varphi(0)>0$. Assume that $J$ is a convex nondecreasing function on $[0,+\infty)$. Then $J$ is continuous on $[0,+\infty)$ and can be extended to $\mathbb{R}$ with preservation of convexity (for instance, by defining $J(t):=J(0)$ for $t<0$ or by means of the even extension). 
Successively using the monotonicity and the Jensen inequality (see, for instance, [12, Sect. 2.2] or Proposition 2), for $f \in C(\mathbb{T})$, we derive from inequality (3.1) that

$$
\begin{aligned}
J\left(\frac{1}{\varphi(0)}\left|A_{\varepsilon, \tau}(f)(t)\right|\right) & \leq J\left(\frac{1}{\varphi(0)} \int_{\mathbb{R}}|f(t+\varepsilon u)| d \mu(u)\right) \\
& \leq \frac{1}{\varphi(0)} \int_{\mathbb{R}} J(|f(t+\varepsilon u)|) d \mu(u), \quad t \in \mathbb{R} .
\end{aligned}
$$

We integrate the left-hand and right-hand sides of inequality (5.1) with respect to $t \in \mathbb{T}$. Applying the Fubini theorem and taking into account the periodicity of $f$, we obtain

$$
\int_{\mathbb{T}} J\left(\frac{1}{\varphi(0)}\left|A_{\varepsilon, \tau}(f)(t)\right|\right) d t \leq \int_{\mathbb{T}} J(|f(t)|) d t .
$$

In view of the arbitrariness of $f$, it is convenient to write the latter inequality in the form

$$
\int_{\mathbb{T}} J\left(\left|A_{\varepsilon, \tau}(f)(t)\right|\right) d t \leq \int_{\mathbb{T}} J(\varphi(0)|f(t)|) d t .
$$

Inequality (5.2) also holds if $\varphi(0)=0$, since, in this case, $\varphi(x) \equiv 0$ and, hence, $A_{\varepsilon, \tau}(f)(t) \equiv 0$ for any $f \in C(\mathbb{T})$. Thus, we obtain the following theorem.

Theorem 4. Assume that $\varphi \in \Phi(\mathbb{R}) \cap C(\mathbb{R}), \tau, \varepsilon \in \mathbb{R}, \varepsilon \neq 0$, and $J$ is a convex nondecreasing function on $[0,+\infty)$. Then:

1) The operator $A_{\varepsilon, \tau}$ generated by the function $\varphi$ by formula (1.1) satisfies inequality (5.2) for any function $f \in C(\mathbb{T})$.

2) If the condition $|\varphi(\varepsilon s-\tau)|=\varphi(0)$ holds for some $s \in \mathbb{Z}$, then equality in (5.2) is attained at the polynomials $f(t)=c e^{i s t}, c \in \mathbb{C}$. If $\tau / \varepsilon \in \mathbb{Z}$, then this condition holds for $s=\tau / \varepsilon$.

If condition (3.4) holds for some $s, m \in \mathbb{Z}, s \neq m$, then equality in (5.2) is attained at the polynomials $f(t)=c e^{i s t}+\nu e^{i m t}, c, \nu \in \mathbb{C}$.

If $\tau \neq 0,|\varphi(-2 \tau)|=\varphi(0), \varepsilon=\tau / n$, and $n \in \mathbb{N}$, then equality in (5.2) is attained at the polynomials $f(t)=c e^{i n t}+\nu e^{-i n t}, c, \nu \in \mathbb{C}$.

3) If the function $J$ is strictly convex at any point of the interval $(0,+\infty)$ and $\varphi(0)>0$, then inequality (5.2) turns into an equality at some function $f \in C(\mathbb{T})$ if and only if, for any $t \in \mathbb{R}$ and $\mu$-almost all $u \in \mathbb{R}$, the equality $e^{-i u \tau} f(t+\varepsilon u)=c(t)$ holds, where $c(t)=A_{\varepsilon, \tau}(f)(t) / \varphi(0) \in C(\mathbb{T})$.

$\mathrm{P} r$ o o f. Only the latter statement needs to be proved. The sufficiency is obvious. Let us prove the necessity. Let inequality (5.2) turn into an equality for some function $f \in C(\mathbb{T})$. Then inequalities (5.1) turn into equalities for all $t \in \mathbb{R}$. Let

$$
\alpha(t):=\frac{1}{\varphi(0)} \int_{\mathbb{R}}|f(t+\varepsilon u)| d \mu(u), \quad t \in \mathbb{R} .
$$

Obviously, $\alpha(t) \geq 0$ for all $t \in \mathbb{R}$. If $\alpha(t)=0$, then $f(t+\varepsilon u)=0$ for $\mu$-almost all $u \in \mathbb{R}$ and, in this case, $c(t)=0$. If $\alpha(t)>0$, then $|f(t+\varepsilon u)|=\alpha(t)$ for $\mu$-almost all $u \in \mathbb{R}$ (see Proposition 2). Since the function $J$ strictly increases on $[0,+\infty)$, inequality (3.1) also turns into an equality for all $t \in \mathbb{R}$. Therefore, for some $\beta(t) \in \mathbb{R}$ and $\mu$-almost all $u \in \mathbb{R}$, we have the equality (see Proposition 1 )

$$
e^{-i u \tau} f(t+\varepsilon u)=e^{i \beta(t)}\left|e^{-i u \tau} f(t+\varepsilon u)\right|=e^{i \beta(t)} \alpha(t)=c(t) .
$$


This implies that $A_{\varepsilon, \tau}(f)(t)=\varphi(0) c(t), t \in \mathbb{R}$.

For $\varepsilon=1 / n, n \in \mathbb{N}$, and $\tau=1$, we can distinguish the case where the condition on the extremal function in Theorem 4 is more clear.

Theorem 5. Let $\varphi(x) \equiv e^{i \beta x} \psi(x)$, where $\beta \in \mathbb{R}$, and let $\psi$ be a 2-periodic function in $\Phi(\mathbb{R}) \cap$ $C(\mathbb{R})$. Let $J$ be a convex nondecreasing function on $[0,+\infty)$. Then the operator $A_{1 / n, 1}, n \in \mathbb{N}$, generated by the function $\varphi$ by formula (1.1) for $\varepsilon=1 / n$ and $\tau=1$ satisfies the inequality

$$
\int_{\mathbb{T}} J\left(\left|A_{1 / n, 1}(f)(t)\right|\right) d t \leq \int_{\mathbb{T}} J(\psi(0)|f(t)|) d t, \quad f \in C(\mathbb{T}) .
$$

Inequality (5.3) turns into an equality, in particular, at every function $f \in C(\mathbb{T})$ whose Fourier series has the form

$$
f(t) \sim \sum_{m \in \mathbb{Z}} d_{m} e^{i n(2 m+1) t}
$$

If the function $J$ is strictly convex at any point of the interval $(0,+\infty)$ and $\psi(0)>0$, then inequality (5.3) turns into an equality at some function $f \in C(\mathbb{T})$ if and only if the functions $(-1)^{s} f\left(t+\frac{\pi s}{n}\right)$ are identical on $\mathbb{R}$ for all $s=0, \ldots, 2 n-1$ such that $\mu_{s}(n, \psi)>0$, where

$$
\mu_{k}(n, \psi)=\sum_{m \in \mathbb{Z}} c_{k+2 n m}(\psi), \quad k \in \mathbb{Z}
$$

and $c_{k}(\psi) \geq 0, k \in \mathbb{Z}$, are the Fourier coefficients of the function $\psi$. If, in addition, the inequalities $\mu_{s}(n, \psi)>0$ and $\mu_{s+1}(n, \psi)>0$ hold for some $s \in \mathbb{Z}$, then inequality (5.3) turns into an equality only at functions $f \in C(\mathbb{T})$ whose Fourier series has the form (5.4).

P r o o f. In our case, $\varphi \in \Phi(\mathbb{R}) \cap C(\mathbb{R})$ and $\varphi(0)=\psi(0)$. Therefore, inequality (5.3) follows immediately from inequality (5.2).

Since the function $\psi$ belongs to $\Phi(\mathbb{R}) \cap C(\mathbb{R})$ and is 2-periodic, its Fourier coefficients $c_{k}(\psi)$, $k \in \mathbb{Z}$, are nonnegative and $\psi$ is expanded into an absolutely convergent Fourier series. Then the function $\varphi$ is also expanded into an absolutely convergent series:

$$
\varphi(x)=\sum_{k \in \mathbb{Z}} c_{k}(\psi) e^{i(\pi k+\beta) x}, \quad x \in \mathbb{R} .
$$

It follows from this representation that the Bochner measure $\mu$ of the function $\varphi$ is concentrated at the points $t_{k}=\pi k+\beta, k \in \mathbb{Z}$, and $\mu\left(\left\{t_{k}\right\}\right)=c_{k}(\psi), k \in \mathbb{Z}$. Therefore, for any $f \in C(\mathbb{T})$, we have

$$
A_{1 / n, 1}(f)(t)=e^{-i \beta} \sum_{k \in \mathbb{Z}}(-1)^{k} f\left(t+\frac{t_{k}}{n}\right) c_{k}(\psi), \quad t \in \mathbb{R} .
$$

Taking into account the periodicity of $f$, it is convenient to divide the terms in this sum into disjoint groups in which the summation index has the form $k+2 n m$ with $m \in \mathbb{Z}$ and $k=0, \ldots, 2 n-1$. Then

$$
A_{1 / n, 1}(f)(t)=e^{-i \beta} \sum_{k=0}^{2 n-1}(-1)^{k} f\left(t+\frac{\pi k+\beta}{n}\right) \mu_{k}(n, \psi), \quad t \in \mathbb{R},
$$

where the numbers $\mu_{k}(n, \psi)$ are defined by formula (5.5). For these numbers, the following equalities hold:

$$
\sum_{k=0}^{2 n-1} \mu_{k}(n, \psi)=\sum_{k \in \mathbb{Z}} c_{k}(\psi)=\psi(0) ; \quad \mu_{k}(n, \psi)=\mu_{k+2 n}(n, \psi), \quad k \in \mathbb{Z} .
$$


If a function $f$ belongs to $C(\mathbb{T})$ and its Fourier series has the form (5.4), then, obviously, $(-1)^{s} f(t+\pi s / n) \equiv f(t)$ for all $s \in \mathbb{Z}$. Therefore, for such functions, we have $A_{1 / n, 1}(f)(t) \equiv$ $e^{-i \beta} \psi(0) f(t+\beta / n)$ and inequality (5.3) turns into an equality.

If the function $J$ is strictly convex at any point of the interval $(0,+\infty)$ and $\psi(0)>0$, then Theorem 4 implies that inequality (5.3) turns into an equality at some function $f \in C(\mathbb{T}) \Longleftrightarrow$ the functions $(-1)^{s} f(t+(\pi s+\beta) / n)$ are identical on $\mathbb{R}$ for all $s \in \mathbb{Z}$ such that $\mu\left(\left\{t_{s}\right\}\right)=c_{s}(\psi)>0$ $\Longleftrightarrow$ the functions $(-1)^{s} f(t+\pi s / n)$ are identical on $\mathbb{R}$ for all $s=0, \ldots, 2 n-1$ such that $\mu_{s}(n, \psi)>0$. The latter equivalence is a consequence of the following properties: (1) the functions of this family with numbers $s \in \mathbb{Z}$ and $s+2 n m, m \in \mathbb{Z}$, are identical; $(2) c_{k}(\psi) \geq 0, \mu_{k}(n, \psi) \geq 0$, $k \in \mathbb{Z}$, and $\mu_{k}(n, \psi)>0 \Longleftrightarrow c_{k+2 n m}(\psi)>0$ for some $m \in \mathbb{Z}$.

Assume that inequality (5.3) turns into an equality at some function $f \in C(\mathbb{T})$. If, in addition, the inequalities $\mu_{s}(n, \psi)>0$ and $\mu_{s+1}(n, \psi)>0$ hold for some $s \in \mathbb{Z}$, then, by what has been proved,

$$
(-1)^{s} f\left(t+\frac{\pi s}{n}\right) \equiv(-1)^{s+1} f\left(t+\frac{\pi(s+1)}{n}\right) .
$$

Then, for the Fourier coefficients of the function $f$, we have the equalities $c_{k}(f)=-e^{i k \pi / n} c_{k}(f)$, $k \in \mathbb{Z}$. If $c_{k}(f) \neq 0$ for some $k \in \mathbb{Z}$, then $k=n(2 m+1)$ for some $m \in \mathbb{Z}$. This means that the Fourier series of the function $f$ has the form (5.4). The theorem is proved.

Remark 4. Let $\varphi(x) \equiv e^{i \beta x} \psi(x)$, where $\beta \in \mathbb{R}$, and assume that a 2-periodic function $\psi$ belongs to $\Phi(\mathbb{R}) \cap C(\mathbb{R})$ and satisfies the inequality $\psi(0)>0$. Then the operator $A_{1 / n, 1}, n \in \mathbb{N}$, satisfies the inequality ( see (5.3) for $J(t)=t^{p}, 1 \leq p<\infty$, or (3.5) for $\varepsilon=1 / n, \tau=1$ )

$$
\left\|A_{1 / n, 1}(f)\right\|_{p} \leqslant \psi(0)\|f\|_{p}, \quad 1 \leqslant p \leqslant \infty, \quad f \in C(\mathbb{T}) .
$$

This inequality turns into an equality, for instance, at every function $f \in C(\mathbb{T})$ whose Fourier series has the form (5.4), since, for such functions, $A_{1 / n, 1}(f)(t) \equiv e^{-i \beta} \psi(0) f(t+\beta / n)$. When $1<p<\infty$, only functions of the form (5.4) are extremal in inequality (5.8) if the inequalities $\mu_{s}(n, \psi)>0$ and $\mu_{s+1}(n, \psi)>0$ hold for some $s \in \mathbb{Z}$ (see Theorem 5 for $J(t)=t^{p}$ ). We state criteria for a function to be extremal when $p=\infty$ and $p=1$. Taking into account Remark 2 and the fact that the Bochner measure $\mu$ of the function $\varphi$ is concentrated at the points $t_{k}=\pi k+\beta, k \in \mathbb{Z}$, and $\mu\left(\left\{t_{k}\right\}\right)=c_{k}(\psi) \geq 0, k \in \mathbb{Z}$ (see the proof of Theorem 5), we obtain:

1) When $p=\infty$, inequality (5.8) turns into an equality at some function $f \in C(\mathbb{T})$ if and only if, for some $\eta, \delta \in \mathbb{R}$, the equality

$$
(-1)^{s} f(\eta+\pi s / n)=e^{i \delta}\|f\|_{\infty}
$$

holds for all $s=0, \ldots, 2 n-1$ such that $\mu_{s}(n, \psi)>0$. This condition is satisfied not only for functions of the form (5.4). For instance, for $s=0, \ldots, 2 n$, we set $f(\pi s / n):=(-1)^{s} M$ and, at the remaining points $t \in[0,2 \pi]$, we define $f$ so that it is continuous on $[0,2 \pi]$ with the only condition $|f(t)| \leq|M|$. For such a function $f$, inequality (5.8) with $p=\infty$ turns into an equality.

If $\mu_{s}(n, \psi)>0$ for $s=0, \ldots, 2 n-1$, then only polynomials of the form $f(t)=c e^{i n t}+\nu e^{-i n t}$, $c, \nu \in \mathbb{C}$, are extremal among trigonometric polynomials of degree at most $n$ for which inequality (5.8) with $p=\infty$ turns into an equality. Indeed, if $f$ is an extremal polynomial of degree at most $n$, then condition (5.9) is satisfied for $s=0, \ldots, 2 n-1$ and, hence, for all $s \in \mathbb{Z}$. Then one can use the arguments of [1, Sect. 84, p. 189] for entire functions in the class $B_{\sigma}$ with $\sigma=n$.

2) When $p=1$, inequality (5.8) turns into an equality at some function $f \in C(\mathbb{T})$ if and only if, for any $t \in \mathbb{R}$, there exists a number $\delta(t) \in \mathbb{R}$ such that the identity

$$
(-1)^{s} f\left(t+\frac{\pi s}{n}\right) \equiv e^{i \delta(t)}\left|f\left(t+\frac{\pi s}{n}\right)\right|
$$


holds for all $s=0, \ldots, 2 n-1$ such that $\mu_{s}(n, \psi)>0$. This implies that if a function $f \in C(\mathbb{T})$ is extremal in inequality (5.8) with $p=1$, then any function of the form $c f(t) g(t)$, where $c \in \mathbb{C}$, $g \in C(\mathbb{T})$, and $g(t) \geq 0$ for all $t \in \mathbb{R}$, is also extremal. In particular, functions of the form $h(t) g(t)$ are extremal if the function $h \in C(\mathbb{T})$ has the form $(5.4), g \in C(\mathbb{T})$, and $g(t) \geq 0$ for all $t \in \mathbb{R}$. In some sense, the converse statement holds: if the inequalities $\mu_{s}(n, \psi)>0$ and $\mu_{s+1}(n, \psi)>0$ hold for some $s \in \mathbb{Z}$, a function $f \in C(\mathbb{T})$ is extremal in inequality (5.8) with $p=1$, and $f(t) \neq 0$ for almost all $t \in \mathbb{R}$ (with respect to the Lebesgue measure), then the function $h(t):=f(t) /|f(t)|$ belongs to $L_{\infty}(\mathbb{T})$ and has the form (5.4) (see the proof of Theorem 5).

We note the following well-known fact. If a function $f \in C(\mathbb{T})$ is extremal in inequality (5.8) with $p=1$, then condition (5.10) implies that the function

$$
g(u):=\int_{\mathbb{T}} f(t+u) e^{-i \delta(t)} d t \in C(\mathbb{T})
$$

is extremal in inequality (5.8) with $p=\infty$. Indeed, for all $s=0, \ldots, 2 n-1$ such that $\mu_{s}(n, \psi)>0$, we have $\|f\|_{1}=(-1)^{s} g(\pi s / n) \leq\|g\|_{\infty} \leq\|f\|_{1}$ and, hence, $(-1)^{s} g(\pi s / n)=\|g\|_{\infty}$.

If $\mu_{s}(n, \psi)>0$ for $s=0, \ldots, 2 n-1$, then only polynomials of the form $f(t)=c e^{i n t}+\nu e^{-i n t}$, $c, \nu \in \mathbb{C}$, are extremal among trigonometric polynomials of degree at most $n$ for which inequality (5.8) with $p=1$ turns into an equality. Indeed, if $f$ is an extremal trigonometric polynomial of degree at most $n$, then condition (5.10) is satisfied for $s=0, \ldots, 2 n-1$. Then one can use the Riesz interpolation formula $[16,17]$ (see also $[28$, Ch. X, Sect. 3, (3.11)])

$$
f^{\prime}\left(t+\frac{\pi}{2 n}\right) \equiv \sum_{s=1}^{2 n}(-1)^{s-1} f\left(t+\frac{\pi s}{n}\right) a_{s}, \text { where all } a_{s}>0 \text { and } \sum_{s=1}^{2 n} a_{s}=n,
$$

which implies the equality $\left\|f^{\prime}\right\|_{1}=n\|f\|_{1}$. Therefore, $f(t)=c e^{i n t}+\nu e^{-i n t}, c, \nu \in \mathbb{C}$ (see [3, Corollary 6]).

Remark 5. If, in Theorem 5, the function $J$ is convex and strictly increasing on $[0,+\infty)$ and $\mu_{s}(n, \psi)>0$ for all $s=0, \ldots, 2 n-1$ (this implies that $\psi(0)>0$ ), then only polynomials of the form $f(t)=c e^{i n t}+\nu e^{-i n t}, c, \nu \in \mathbb{C}$, are extremal among trigonometric polynomials of degree at most $n$ for which inequality (5.3) turns into an equality. Indeed, if inequality (5.3) turns into an equality at some function $f \in C(\mathbb{T})$, then the corresponding inequalities (5.1) and (3.1) turn into equalities for any $t \in \mathbb{R}$ and, hence, inequality (5.8) with $p=1$ turns into an equality at $f$. Then we need to use the last statement in Remark 4.

In conclusion of this section, we note that the integral inequalities (5.2) for the class of trigonometric polynomials and for different differential operators and Szegő compositions were studied by many authors, in particular, by A. Zygmund, V.V. Arestov, V.I. Ivanov, E.A. Storozhenko, V.G. Krotov, P. Oswald, and A.I. Kozko. In this case, not only convex functions $J$ were considered. A history of this question was described in great detail in the paper by Arestov [4].

\section{Generalization of Bernstein-Szegő inequalities}

We denote by $\mathscr{F}_{n}, n \in \mathbb{N}$, the set of trigonometric polynomials

$$
f(t):=\sum_{|k| \leq n} c_{k} e^{i k t}=\frac{a_{0}}{2}+\sum_{k=1}^{n}\left(a_{k} \cos k t+b_{k} \sin k t\right), \quad c_{k}=c_{k}(f) \in \mathbb{C},
$$


of degree at most $n$ with coefficients in $\mathbb{C}$, where $a_{k}:=c_{k}+c_{-k}$ and $b_{k}:=i\left(c_{k}-c_{-k}\right), k \geq 0$. There are several different definitions of fractional derivative. The following operator for $r>0$ and $\beta \in \mathbb{R}$ presumably first appeared in the paper by Sz.-Nagy [21, equality (2) for $m=1, \lambda(k)=k^{r}$ ]. For $f \in \mathscr{F}_{n}$, we define

$$
f^{(r, \beta)}(t):=\sum_{|k| \leq n}|k|^{r} e^{i \beta \operatorname{sign} k} c_{k} e^{i k t}=\sum_{k=1}^{n} k^{r}\left(a_{k} \cos (k t+\beta)+b_{k} \sin (k t+\beta)\right) .
$$

For $\beta=r \pi / 2$, we obtain the Weyl derivative which, for $r \in \mathbb{N}$, coincides with the usual derivative of order $r$. Often, this operator is called the Weyl-Nagy derivative.

Let $J$ be a convex and nondecreasing function on $[0,+\infty)$. Kozko proved (see [11, Theorem 1 , Corollary 1]) that if $1 \leq p \leq \infty$, then, for any $n \in \mathbb{N}, r \geq 1$, and $\beta \in \mathbb{R}$, the following inequalities hold:

$$
\begin{gathered}
\int_{\mathbb{T}} J\left(\left|f^{(r, \beta)}(f)(t)\right|\right) d t \leq \int_{\mathbb{T}} J\left(n^{r}|f(t)|\right) d t, \quad f \in \mathscr{F}_{n}, \\
\left\|f^{(r, \beta)}\right\|_{p} \leq n^{r}\|f\|_{p}, \quad f \in \mathscr{F}_{n} .
\end{gathered}
$$

For the usual derivative, i.e., when $r=1$ and $\beta=\pi / 2$, inequality (6.2) was proved by Bernstein in the case $p=\infty$. For $r=1$ and $\beta \in \mathbb{R}$, inequality (6.2) was obtained by Szegő [20] in the case $p=\infty$ and inequality (6.1) was proved by Zygmund [28, Ch. X, Sect. 3, (3.25)] (his proof for real polynomials is also true for polynomials in $\mathscr{F}_{n}$ ). This and the identity

$$
f^{(r+1, \beta)}(t) \equiv\left(f^{(r, \beta)}(t)\right)^{(1,0)}, \quad r>0, \quad \beta \in \mathbb{R},
$$

imply the validity of inequality (6.2) for any $r \in \mathbb{N}$. Inequality (6.2) for $p=\infty, r \geq 1, \beta=-r \pi / 2$, and $\beta=0$ (the case of the Riesz derivative) was proved by Lizorkin [13, Theorems $\left.2,2^{\prime}\right]$.

Obviously, inequalities (6.1) and (6.2) turn into equalities for the polynomials $f(t)=c e^{i n t}+$ $\nu e^{-i n t}, c, \nu \in \mathbb{C}$. Szegö $[20$, p. 66] proved that, in inequality $(6.2)$ with $p=\infty$, there are no other extremal polynomials in the case $r=1$ and $\beta \neq q \pi, q \in \mathbb{Z}$ (see also arguments in [1, Sect. 84, p. 189]). If, in addition, the function $t J^{\prime}(t)$ is strictly increasing on $(0,+\infty)$, then, in inequalities (6.1) and (6.2) for $1 \leq p<\infty, n \in \mathbb{N}, r \geq 1$, and $\beta \in \mathbb{R}$, there are no other extremal polynomials at least in the following cases (see [3, Corollary 6], [5, Theorems 1,2]): (1) in the case of the usual derivative of order $r \in \mathbb{N}$; (2) $n=1, r \geq 1$, and $\beta \in \mathbb{R}$ or $n \geq 2, r \geq \ln (2 n) / \ln (n /(n-1))$, and $\beta \in \mathbb{R}$.

For $r=1$ and $\beta \neq q \pi, q \in \mathbb{Z}$, in inequalities (6.2) and (6.1) (if, in addition, the function $J(t)$ is strictly increasing on $(0,+\infty)$ ), only polynomials of the form $f(t)=a \cos n t+b \sin n t, a, b \in \mathbb{R}$, are extremal in the class of real trigonometric polynomials. This result is due to Zygmund [28, Ch. X, Sect 3, (3.24), (3.25)].

Other cases in which inequality (6.2) holds, when $r<1$ or $0 \leq p<1$, were considered in the paper by Arestov and Glazyrina [5], where these inequalities are called Bernstein-Szegő inequalities and a complete history of such inequalities is given.

Inequalities more general than (6.1) and (6.2) are obtained from Theorem 5 under an appropriate choice of the function $\psi$. The method of construction of the function $\psi$ described below is essentially contained in the paper by Lizorkin [13].

Assume that $g \in \Phi(\mathbb{R}) \cap C(\mathbb{R})$, supp $g \subset[-1,1]$, and $\beta \in \mathbb{R}$. We consider the auxiliary function $F(x):=g(-x) e^{-i \beta x}, x \in \mathbb{R}$. Obviously, $F \in \Phi(\mathbb{R}) \cap C(\mathbb{R})$ and supp $F \subset[-1,1]$. Using the function $F$, we construct the 2-periodic function $\psi \in \Phi(\mathbb{R}) \cap C(\mathbb{R})$ (see Corollary 1). For $x \in[-2,2]$, we have

$$
\psi(x-1)=F(x-1)+F(x+1)=g(1-x) e^{-i \beta(x-1)}+g(-1-x) e^{-i \beta(x+1)} .
$$


Then

$$
\psi(x-1)=e^{-i \beta x}\left\{\begin{array}{lr}
g(1-|x|) e^{i \beta}, & 0 \leq x \leq 2 \\
g(|x|-1) e^{-i \beta}, & -2 \leq x \leq 0 .
\end{array}\right.
$$

Taking into account that the real and imaginary parts of a positive definite function are even and odd functions, respectively, we obtain the equality $\psi(x-1)=e^{-i \beta x} e^{i \beta \operatorname{sign} x} g_{0}(x),|x| \leq 2$, where

$$
g_{0}(x)=\operatorname{Re} g(1-|x|)+i \operatorname{sign} x \operatorname{Im} g(1-|x|), \quad|x| \leq 2 .
$$

Obviously, the function $\varphi(x):=e^{i \beta x} \psi(x)$ belongs to $\Phi(\mathbb{R}) \cap C(\mathbb{R})$ and

$$
\varphi(x-1)=e^{-i \beta} g_{0}(x) e^{i \beta \operatorname{sign} x}=e^{-i \beta}(\operatorname{Re} g(1-|x|)+i \operatorname{sign} x \operatorname{Im} g(1-|x|)) e^{i \beta \operatorname{sign} x}, \quad|x| \leq 2 .
$$

Consider the operator $A_{1 / n, 1}$ generated by the function $\varphi$ by formula (1.1) for $\varepsilon=1 / n$ and $\tau=1$. We can apply Theorem 5 and Remarks 4 and 5 to this operator. It should be taken into account that $\psi(0)=g(0)$ and $c_{k}(\psi)=\widehat{g}(-\beta-k \pi) / 2, k \in \mathbb{Z}$. For polynomials $f \in \mathscr{F}_{2 n}$, the operator $A_{1 / n, 1}$ has the following form (see (3.2) and (6.3)):

$$
A_{1 / n, 1}(f)(t) \equiv e^{-i \beta} \sum_{|k| \leq 2 n}\left(\operatorname{Re} g\left(1-\frac{|k|}{n}\right)+i \operatorname{sign} k \operatorname{Im} g\left(1-\frac{|k|}{n}\right)\right) e^{i \beta \operatorname{sign} k} c_{k}(f) e^{i k t}
$$

We introduce one more parameter. Obviously, for any $\theta \in[-1,1]$, the function

$$
g_{\theta}(x):=((1+\theta) g(x)+(1-\theta) g(-x)) / 2=\operatorname{Re} g(x)+i \theta \operatorname{Im} g(x), \quad x \in \mathbb{R},
$$

also belongs to the class $\Phi(\mathbb{R}) \cap C(\mathbb{R})$ and $\operatorname{supp} g_{\theta} \subset[-1,1]$. Therefore, all the above arguments are applicable to the function $g_{\theta}$ as well. It should be taken into account that, for the corresponding function $\psi_{\theta}$, we have $\psi_{\theta}(0)=g_{\theta}(0)=g(0)$ and

$$
c_{k}\left(\psi_{\theta}\right)=((1+\theta) \widehat{g}(-\beta-k \pi)+(1-\theta) \widehat{g}(\beta+k \pi)) / 4, \quad k \in \mathbb{Z} .
$$

For the function $\varphi_{\theta}(x):=e^{i \beta x} \psi_{\theta}(x) \in \Phi(\mathbb{R}) \cap C(\mathbb{R})$, we consider the corresponding operator $A_{1 / n, 1}$ with $\varepsilon=1 / n$ and $\tau=1$ (see (1.1)). We state the results obtained in Theorem 5 and Remarks 4 and 5 for the following operator defined on polynomials $f \in \mathscr{F}_{2 n}$ :

$$
\begin{aligned}
& D_{n, \theta}^{g, \beta}(f)(t):=A_{1 / n, 1}(f)(t) \equiv \\
& e^{-i \beta} \sum_{|k| \leq 2 n}\left(\operatorname{Re} g\left(1-\frac{|k|}{n}\right)+i \theta \operatorname{sign} k \operatorname{Im} g\left(1-\frac{|k|}{n}\right)\right) e^{i \beta \operatorname{sign} k} c_{k}(f) e^{i k t} .
\end{aligned}
$$

Theorem 6. Assume that $g \in \Phi(\mathbb{R}) \cap C(\mathbb{R})$, supp $g \subset[-1,1], g(0)>0, \beta \in \mathbb{R}, \theta \in[-1,1]$, and $1 \leq p \leq \infty$. Let $J$ be a convex nondecreasing function on $[0,+\infty)$. Then:

1) For any $n \in \mathbb{N}$, we have

$$
\begin{gathered}
\int_{\mathbb{T}} J\left(\left|D_{n, \theta}^{g, \beta}(f)(t)\right|\right) d t \leq \int_{\mathbb{T}} J(g(0)|f(t)|) d t, \quad f \in \mathscr{F}_{2 n}, \\
\left\|D_{n, \theta}^{g, \beta}(f)\right\|_{p} \leq g(0)\|f\|_{p}, \quad f \in \mathscr{F}_{2 n} .
\end{gathered}
$$

Inequalities (6.5) and (6.6) turn into equalities, for instance, for polynomials of the form $f(t)=$ $c e^{i n t}+\nu e^{-i n t}, c, \nu \in \mathbb{C}$.

2) If the function $J$ is strictly convex at any point of the interval $(0,+\infty)$, then inequality (6.5) or inequality (6.6) with $p \in(1, \infty)$ turns into an equality at some polynomial $f \in \mathscr{F}_{2 n}$ if and only if the 
functions $(-1)^{s} f(t+\pi s / n)$ are identical on $\mathbb{R}$ for all $s=0, \ldots, 2 n-1$ such that $\mu_{s}(n, g, \beta, \theta)>0$, where, for $k \in \mathbb{Z}$,

$$
4 \mu_{k}(n, g, \beta, \theta)=(1+\theta) \sum_{m \in \mathbb{Z}} \widehat{g}(-\beta-(k+2 n m) \pi)+(1-\theta) \sum_{m \in \mathbb{Z}} \widehat{g}(\beta+(k+2 n m) \pi) .
$$

If, in addition, for some $s \in \mathbb{Z}$, the inequalities $\mu_{s}(n, g, \beta, \theta)>0$ and $\mu_{s+1}(n, g, \beta, \theta)>0$ hold, then inequality $(6.5)$ or inequality $(6.6)$ with $p \in(1, \infty)$ turns into an equality only at the polynomials $f(t)=c e^{i n t}+\nu e^{-i n t}, c, \nu \in \mathbb{C}$.

3) When $p=\infty$, inequality (6.6) turns into an equality at some polynomial $f \in \mathscr{F}_{2 n}$ if and only if, for some $\eta, \delta \in \mathbb{R}$, the equality $(-1)^{s} f(\eta+\pi s / n)=e^{i \delta}\|f\|_{\infty}$ holds for all $s=0, \ldots, 2 n-1$ such that $\mu_{s}(n, g, \beta, \theta)>0$.

If $\mu_{s}(n, g, \beta, \theta)>0$ for $s=0, \ldots, 2 n-1$, then only polynomials of the form $f(t)=c e^{i n t}+$ $\nu e^{-i n t}, c, \nu \in \mathbb{C}$, are extremal among trigonometric polynomials of degree at most $n$ for which inequality (6.6) with $p=\infty$ turns into an equality.

4) When $p=1$, inequality (6.6) turns into an equality at some polynomial $f \in \mathscr{F}_{2 n}$ if and only if, for any $t \in \mathbb{R}$, there exists a number $\delta(t) \in \mathbb{R}$ such that the identity $(-1)^{s} f(t+\pi s / n) \equiv$ $e^{i \delta(t)}|f(t+\pi s / n)|$ holds for all $s=0, \ldots, 2 n-1$ such that $\mu_{s}(n, g, \beta, \theta)>0$.

If a polynomial $f \in \mathscr{F}_{q}, 1 \leq q<2 n$, is extremal in inequality (6.6) with $p=1$, then any polynomial of the form $c f(t) g(t)$, where $c \in \mathbb{C}, g \in \mathscr{F}_{2 n-q}$, and $g(t) \geq 0$ for all $t \in \mathbb{R}$, is also extremal. In particular, polynomials of the form $\left(c e^{i n t}+\nu e^{-i n t}\right) g(t)$, where $c, \nu \in \mathbb{C}$ and $g$ is an arbitrary nonnegative trigonometric polynomial of degree at most n, are extremal in inequality (6.6) with $p=1$.

5) If $\mu_{s}(n, g, \beta, \theta)>0$ for all $s=0, \ldots, 2 n-1$ and the function $J$ is strictly increasing on $(0,+\infty)$, then only polynomials of the form $f(t)=c e^{i n t}+\nu e^{-i n t}, c, \nu \in \mathbb{C}$, are extremal among trigonometric polynomials of degree at most $n$ for which inequality (6.5) or inequality (6.6) with $p=1$ turns into an equality.

Remark 6. If $q \in \mathbb{Z}$ and $q=2 n l+r$, where $l, r \in \mathbb{Z}$ and $0 \leq r \leq 2 n-1$, then

$$
\mu_{k}(n, g, \beta+\pi q, \theta)=\left\{\begin{array}{lc}
\mu_{k+r}(n, g, \beta, \theta), & 0 \leq k \leq 2 n-1-r \\
\mu_{k+r-2 n}(n, g, \beta, \theta), & 2 n-r \leq k \leq 2 n-1, \quad r \geq 1 .
\end{array}\right.
$$

Remark 7. Inequalities (6.1) and (6.2) follow from inequalities (6.5) and (6.6) if, for $g$, we take the function $g_{r}(x)=(1-|x|)_{+}^{r}$ which is positive definite for $r \geq 1$ (the Pólya property). Since $g_{r}(1-|x|)=|x|^{r}$ for $|x| \leq 1$, we have $D_{n, \theta}^{g_{r}, \beta}(f)(t) \equiv e^{-i \beta} f^{(r, \beta)}(t) / n^{r}$ for any polynomial $f \in \mathscr{F}_{n}$, $n \in \mathbb{N}$. In our case, the values (6.7) are independent of $\theta$ and such that

$$
\left.\mu_{k}\left(n, g_{r}, \beta\right)=\sum_{m \in \mathbb{Z}} \widehat{g_{r}}(\beta+(k+2 n m) \pi)\right) / 2, \quad k \in \mathbb{Z} .
$$

It is well known that, for $r>1$, the Fourier transform $\widehat{g_{r}}(t)$ is positive for all $t \in \mathbb{R}$ (see, for instance, $[27$, Lemma $7, n=\lambda=\delta=1])$. Therefore, $\mu_{s}\left(n, g_{r}, \beta\right)>0$ for all $r>1, \beta \in \mathbb{R}, n \in \mathbb{N}$, and $s \in \mathbb{Z}$.

For $r=1$, the Fourier transform of the function $g_{1}$ is easily calculated and is equal to $\widehat{g_{1}}(t)=$ $2(1-\cos t) / t^{2}$. Obviously, $\widehat{g_{1}}(t)=0$ only for $t=2 q \pi$ with $q \in \mathbb{Z}, q \neq 0$. Therefore, if $\beta \neq q \pi$, $q \in \mathbb{Z}$, then $\mu_{s}\left(n, g_{1}, \beta\right)>0$ for all $n \in \mathbb{N}$ and $s \in \mathbb{Z}$.

If $\beta=0$ and $n \in \mathbb{N}$, then: (1) $\mu_{s}\left(n, g_{1}, 0\right)>0$ for $s=0$ and for all odd $s \in[1,2 n-1]$; (2) $\mu_{s}\left(n, g_{1}, 0\right)=0$ for all even $s \in[2,2 n-1]$ if $n \geq 2$. In this case, the number of positive values 
among $\mu_{s}\left(n, g_{1}, 0\right), s=0, \ldots, 2 n-1$, is $n+1$ and the remaining are zero. The latter property also holds for any $\beta=\pi q$ with $q \in \mathbb{Z}$ (see Remark 6).

Thus, only polynomials of the form $f(t)=c e^{i n t}+\nu e^{-i n t}, c, \nu \in \mathbb{C}$, are extremal in inequalities $(6.1)$ and (6.2) under conditions $\left(A_{1}\right)$ and $\left(B_{1}\right)$ or $\left(A_{2}\right)$ and $\left(B_{2}\right)$, where

$\left(A_{1}\right) r>1$ and $\beta \in \mathbb{R}$; or $r=1, \beta \in \mathbb{R}$, and $n=1$; or $r=1, \beta \neq \pi q, q \in \mathbb{Z}$, and $n \geq 2$;

$\left(B_{1}\right)$ the function $J$ is strictly increasing on $(0,+\infty)$ for $(6.1)$ or $1 \leq p \leq \infty$ for $(6.2)$;

$\left(A_{2}\right) r=1, \beta=\pi q$ with $q \in \mathbb{Z}$, and $n \geq 2$;

$\left(B_{2}\right)$ the function $J$ is strictly convex on $(0,+\infty)$ for $(6.1)$ or $1<p<\infty$ for (6.2).

The case where $r=1, \beta=\pi q$ with $q \in \mathbb{Z}, n \geq 2$, and $p=1$ or $p=\infty$ has not been studied.

\section{Case of piecewise linear functions}

In [15], the following R.M. Trigub problem on the positive definiteness of piecewise linear functions was solved. For given $\alpha \in(0,1)$ and $h \in \mathbb{R}$, the function $f_{\alpha, h}: \mathbb{R} \rightarrow \mathbb{C}$ is defined as follows: (1) the function $f_{\alpha, h}$ is even; (2) $f_{\alpha, h}(x)=0$ for $x>1$, the function $f_{\alpha, h}$ is linear on each of the intervals $[0, \alpha]$ and $[\alpha, 1], f_{\alpha, h}(0)=1, f_{\alpha, h}(\alpha)=h$, and $f_{\alpha, h}(1)=0$. For any fixed $\alpha \in(0,1)$, it is required to find the set of all $h \in \mathbb{R}$ such that the piecewise linear function $f_{\alpha, h}$ is positive definite on $\mathbb{R}$. If $0 \leq h \leq 1-\alpha$, then the continuous even function $f_{\alpha, h}(x)$ is convex on $(0,+\infty), f_{\alpha, h}(+\infty)=0$, and, hence, it is positive definite by the Pólya theorem (see, for instance, [14, Theorem 4.3.1]). A complete description of such $h \in \mathbb{R}$ is given in the following theorem.

Theorem 7 [15]. Let $\alpha \in(0,1)$ and $h \in \mathbb{R}$. Then $f_{\alpha, h} \in \Phi(\mathbb{R})$ if and only if $m(\alpha) \leq h \leq 1-\alpha$, where $m(\alpha)=0$ if $1 / \alpha \notin \mathbb{N}$ and $m(\alpha)=-\alpha$ if $1 / \alpha \in \mathbb{N}$.

From Theorem 7, we obtain the following sufficient condition for the positive definiteness.

Corollary 2. If a function $g \in C(\mathbb{R})$ is even, nonnegative, decreasing, and convex on $(0,+\infty)$, then, for $\alpha \in(0,1), 1 / \alpha \in \mathbb{N}$, and $-\alpha \leq h \leq 1-\alpha$, the function $g_{\alpha, h}(x):=h g(x)+(1-\alpha-h) g(x / \alpha)$ belongs to the class $\Phi(\mathbb{R})$.

The nontrivial case here is when $-\alpha \leq h<0$.

$\mathrm{P}$ r o o f. The function $g$ is represented in the form (see, for instance, [26])

$$
g(x)=\int_{0}^{+\infty}(1-|s x|)_{+} d \mu(s), \quad x \in \mathbb{R},
$$

where $\mu$ is a nonnegative finite Borel measure on $[0,+\infty)$. Obviously,

$$
g_{\alpha, h}(x)=(1-\alpha) \int_{0}^{+\infty} f_{\alpha, h}(s x) d \mu(s), \quad x \in \mathbb{R} .
$$

For the specified $\alpha$ and $h$, we have $f_{\alpha, h} \in \Phi(\mathbb{R})$. Hence, $g_{\alpha, h} \in \Phi(\mathbb{R})$ (see, for instance, $[27$, Lemma 1]).

One can use the positive definite function $g_{\alpha, h}$ given in Corollary 2 to obtain new sharp inequalities for trigonometric polynomials. 
Let a function $g \in C(\mathbb{R})$ be even, nonnegative, decreasing, and convex on $(0,+\infty)$, and let $\operatorname{supp} g \subset[-1,1]$. Assume that $n \in \mathbb{N}, n \geq 2$, and $-1 / n \leq h \leq 1-1 / n$. Let $g_{1 / n, h}(x):=$ $h g(x)+(1-1 / n-h) g(n x), x \in \mathbb{R}$. It follows from Corollary 2 that $g_{1 / n, h} \in \Phi(\mathbb{R}) \cap C(\mathbb{R})$. Since $\operatorname{supp} g \subset[-1,1]$, we have $\operatorname{supp} g_{1 / n, h} \subset[-1,1]$. Therefore, for the function $g_{1 / n, h}$, we can construct operator (6.4) which does not depend on the parameter $\theta$, since $\operatorname{Im}\left(g_{1 / n, h}\right) \equiv 0$. It is not difficult to verify that, for polynomials $f \in \mathscr{F}_{2 n}$, the following equality holds:

$$
D_{n, 0}^{g_{1 / n, h}, \beta}(f)(t)=h D_{n, 0}^{g, \beta}(f)(t)+(1-1 / n-h) g(0) R_{n}^{\beta}(f)(t),
$$

where

$$
R_{n}^{\beta}(f)(t):=e^{-i \beta} \sum_{|k|=n} e^{i \beta \operatorname{sign} k} c_{k}(f) e^{i k t}=\frac{e^{-i \beta}}{\pi} \int_{-\pi}^{\pi} \cos (n x-\beta) f(t+x) d x .
$$

We note that $g_{1 / n, h}(0)=(1-1 / n) g(0)$. In addition, if $g(x)=(1-|x|)_{+}^{r}, r \geq 1$, then $D_{n, \theta}^{g_{r}, \beta}(f)(t) \equiv$ $e^{-i \beta} f^{(r, \beta)}(t) / n^{r}$ for any polynomial $f \in \mathscr{F}_{n}$. We write Theorem 6 for the operator (7.1) and restrict ourselves only to inequality (6.6).

Theorem 8. Let a function $g \in C(\mathbb{R})$ be even, nonnegative, decreasing, and convex on $(0,+\infty)$, and let $\operatorname{supp} g \subset[-1,1]$. Assume that $n \geqslant 2,-1 / n \leq h \leq 1-1 / n, \beta \in \mathbb{R}$, and $1 \leqslant p \leqslant \infty$. Then, for any polynomial $f \in \mathscr{F}_{2 n}$, we have

$$
\left\|h D_{n, 0}^{g, \beta}(f)+(1-1 / n-h) g(0) R_{n}^{\beta}(f)\right\|_{p} \leqslant(1-1 / n) g(0)\|f\|_{p} .
$$

If $r \geq 1$, then, for any polynomial $f \in \mathscr{F}_{n}$, we have

$$
\left\|h f^{(r, \beta)} / n^{r}+(1-1 / n-h) e^{i \beta} R_{n}^{\beta}(f)\right\|_{p} \leqslant(1-1 / n)\|f\|_{p} .
$$

Inequalities (7.3) and (7.4) turn into equalities for polynomials of the form $f(t)=c e^{i n t}+\nu e^{-i n t}$, $c, \nu \in \mathbb{C}$.

Without proof, we note that if the function $g$ in Theorem 8 is not piecewise linear on $[0,+\infty)$ with equidistant nodes, then only polynomials of the form $f(t)=c e^{i n t}+\nu e^{-i n t}, c, \nu \in \mathbb{C}$, are extremal in inequality (7.3) with $p \in(1, \infty)$. When $p=1$ or $p=\infty$, a similar conclusion holds, but for the class of trigonometric polynomials of degree at most $n$. If $r>1$, then only polynomials of the form $f(t)=c e^{i n t}+\nu e^{-i n t}, c, \nu \in \mathbb{C}$, are extremal in inequality (7.4).

\section{Interpolation formulas for periodic functions}

If the trigonometric series on the right-hand side of relation (3.2) converges uniformly on $\mathbb{T}$, then one can put the sign of equality in this relation and the obtained equality can be regarded as some interpolation formula. We explain this with the example of the following theorem.

Theorem 9. Assume that $n \in \mathbb{N}$, a 2-periodic function $\psi$ belongs to $\Phi(\mathbb{R}) \cap C(\mathbb{R}), \beta \in \mathbb{R}$, and the numbers $\mu_{k}(n, \psi)$ are defined by formula (5.5). Then the identity

$$
\sum_{k \in \mathbb{Z}} e^{i \beta k / n} \psi\left(\frac{k}{n}-1\right) c_{k}(f) e^{i k t} \equiv \sum_{k=0}^{2 n-1}(-1)^{k} f\left(t+\frac{\pi k+\beta}{n}\right) \mu_{k}(n, \psi)
$$

holds for any function $f \in C(\mathbb{T})$ such that the series on the left converges uniformly on $\mathbb{T}$. Moreover, $\mu_{0}(n, \psi)+\ldots+\mu_{2 n-1}(n, \psi)=\psi(0), c_{k}(\psi) \geq 0, k \in \mathbb{Z}, \mu_{k}(n, \psi) \geq 0, k=0, \ldots, 2 n-1$, and $\mu_{k}(n, \psi)=0$ for some $k=0, \ldots, 2 n-1$ if and only if $c_{k+2 n m}(\psi)=0$ for all $m \in \mathbb{Z}$. 
P r o o f. Consider operator (1.1) for the function $\varphi(x) \equiv e^{i \beta x} \psi(x)$. Under the conditions of the theorem, we can put the sign of equality in relation (3.2) for $\varepsilon=1 / n$ and $\tau=1$. Therefore, the left-hand side of equality (5.6) can be replaced by the sum of the series in (3.2). We obtain identity (8.1) with accuracy up to the factor $e^{-i \beta}$. The specified properties of the numbers $\mu_{k}(n, \psi)$ follow from (5.5) and (5.7).

Corollary 3. Assume that $g \in \Phi(\mathbb{R}) \cap C(\mathbb{R})$, supp $g \subset[-1,1], \beta \in \mathbb{R}$, and $n \in \mathbb{N}$. Then, for any polynomial $f \in \mathscr{F}_{2 n}$, the following equality holds:

$$
\begin{aligned}
& \sum_{|k| \leq 2 n}\left(\operatorname{Re} g\left(1-\frac{|k|}{n}\right)+i \operatorname{sign} k \operatorname{Im} g\left(1-\frac{|k|}{n}\right)\right) e^{i \beta \operatorname{sign} k} c_{k}(f) e^{i k t} \\
& =\sum_{k=0}^{2 n-1}(-1)^{k} f\left(t+\frac{\pi k+\beta}{n}\right) \mu_{k}(n, g, \beta), \quad t \in \mathbb{R},
\end{aligned}
$$

where $\mu_{k}(n, g, \beta)=\sum_{m \in \mathbb{Z}} \widehat{g}(-\beta-(k+2 n m) \pi) / 2, k \in \mathbb{Z}$, and $\sum_{k=0}^{2 n-1} \mu_{k}(n, g, \beta)=g(0)$.

P r o o f. Let $\psi$ be a 2-periodic function, and let $\psi(x)=g(-x) e^{-i \beta x}$ for $x \in[-1,1]$. Then $\psi \in \Phi(\mathbb{R}) \cap C(\mathbb{R})$ and

$$
\psi(x-1)=e^{-i \beta x} e^{i \beta \operatorname{sign} x}(\operatorname{Re} g(1-|x|)+i \operatorname{sign} x \operatorname{Im} g(1-|x|)), \quad|x| \leq 2 .
$$

It remains to take into account that $c_{k}(\psi)=\widehat{g}(-\beta-k \pi) / 2, k \in \mathbb{Z}$.

Remark 8. We note that if, for $g$, we take the function $g_{r}(x)=(1-|x|)_{+}^{r}, r \geq 1$, then, in (8.2), we obtain the interpolation formula of A.I. Kozko [11] (and of M. Riesz and of G. Szegő for $r=1$ ) for the Weyl-Nagy derivative:

$$
f^{(r, \beta)}(t)=n^{r} \sum_{k=0}^{2 n-1}(-1)^{k} f\left(t+\frac{\pi k+\beta}{n}\right) \mu_{k}\left(n, g_{r}, \beta\right), \quad t \in \mathbb{R}, \quad f \in \mathscr{F}_{n} ; \quad \sum_{k=0}^{2 n-1} \mu_{k}\left(n, g_{r}, \beta\right)=1,
$$

where $\mu_{k}\left(n, g_{r}, \beta\right)>0$ for all $n \in \mathbb{N}, k=0, \ldots, 2 n-1, \beta \in \mathbb{R}$, and $r>1$. These coefficients are also positive for $r=1$ if $n=1$ and $\beta \in \mathbb{R}$ or if $n \geq 2$ and $\beta \neq q \pi, q \in \mathbb{Z}$. If $r=1, n \geq 2$, and $\beta=\pi q$ with $q \in \mathbb{Z}$, then, the number of positive coefficients among $\mu_{k}\left(n, g_{1}, \beta\right), k=0, \ldots, 2 n-1$, is $n+1$ and the remaining are zero (see Remark 7 ). For $r=1$, these coefficients are easily calculated. Since $\widehat{g_{1}}(t)=2(1-\cos t) / t^{2}$, we have

$$
\mu_{k}\left(n, g_{1}, \beta\right)=\frac{1-(-1)^{k} \cos \beta}{4 n^{2}} \sum_{m \in \mathbb{Z}} \frac{1}{\left(\frac{\beta+k \pi}{2 n}+m \pi\right)^{2}}=\frac{1-(-1)^{k} \cos \beta}{2 n^{2}\left(1-\cos \frac{\beta+k \pi}{n}\right)}>0, \quad \beta \neq q \pi, \quad q \in \mathbb{Z},
$$

For $\beta=q \pi$ with $q \in \mathbb{Z}$, we can restrict ourselves to the case $\beta=0$ (see Remark 6$): \mu_{2 k}\left(n, g_{1}, 0\right)=0$ for $k=1, \ldots, n-1$ (if $n \geq 2), \mu_{0}\left(n, g_{1}, 0\right)=1 / 2$, and

$$
\mu_{2 k-1}\left(n, g_{1}, 0\right)=\frac{1}{n^{2}\left(1-\cos \frac{(2 k-1) \pi}{n}\right)}>0, \quad k=1, \ldots, n .
$$


Remark 9. It is not difficult to see that all the arguments in the proof of Theorem 9 remain in force also in the case where the 2-periodic continuous function $\psi$ is expanded in an absolutely convergent Fourier series (without the assumption of nonnegativity of the Fourier coefficients $c_{k}(\psi)$ ). Therefore, the following statement holds: Assume that a 2-periodic function $\psi \in C(\mathbb{R})$ is expanded into an absolutely convergent Fourier series and $\beta \in \mathbb{R}$. Then equality (8.1) holds for any function $f \in C(\mathbb{T})$ such that the series on the left in (8.1) converges uniformly on $\mathbb{T}$.

\section{Conclusion}

In conclusion, we point out some problems which, in our opinion, have not been solved yet.

1) To prove or disprove that only polynomials of the form $f(t)=c e^{i n t}+\nu e^{-i n t}, c, \nu \in \mathbb{C}$, are extremal in the Berstein-Szegö inequality (6.2) for $r=1$ and $\beta=0$ (the case of the derivative of the adjoint polynomial) when $p=\infty$ or $p=1$. When $p=\infty$, this case was distinguished in the paper by Szegö $[20$, p. 66$]$. We note that the arguments in the monographs by Zygmund [28, Ch. X, Sect. 3, (3.24)] and Akhiezer [1, Sect. 84, p. 189] corresponding to this case are not correct, since some coefficients in the interpolation formulas are zero (see [28, Ch. X, Sect. 3, (3.22)] for $\alpha=\pi / 2$ and [1, Sect. 84, p. 188, (II)] for $\alpha=0$ ).

2) Let $n \in \mathbb{N}$, and let, for a trigonometric polynomial $f \in \mathscr{F}_{n}$, condition (5.9) or (5.10) be satisfied for all integers $s=0, \ldots, 2 n-1$. Then $f(t)=c e^{i n t}+\nu e^{-i n t}, c, \nu \in \mathbb{C}$ (see Remark 4). The question is, which values of $s$ can be left to have the same conclusion? This is a more general problem than the previous one.

3) To prove or disprove that if, for some $s \in \mathbb{Z}$, inequalities $\mu_{s}(n, \psi)>0$ and $\mu_{s+1}(n, \psi)>0$ hold and a function $f \in C(\mathbb{T})$ is extremal in inequality (5.8) with $p=1$, then $f(t)=h(t) g(t)$, where the function $h$ belongs to $L_{\infty}(\mathbb{T})$ and has the form (5.4), $g \in C(\mathbb{T})$, and $g(t) \geq 0$ for $t \in \mathbb{R}$. This is true if, in addition, $f(t) \neq 0$ for almost all $t \in \mathbb{R}$ with respect to the Lebesgue measure (see Remark 4 for the case $p=1$ ).

\section{Acknowledgements}

I express my gratitude to the Organizing Committee of the International S.B. Stechkin Summer Workshop-Conference on Function Theory for the given opportunity to participate in this event and to all participants for creating a warm and working atmosphere. In addition, I would like to thank Professor V.V. Arestov and P.Yu. Glazyrina for useful discussions and sharing information and Professor A.A. Kovalevskii for the translation of my article into English.

\section{REFERENCES}

1. Akhiezer N.I. Lectures on approximation theory. Moscow: Nauka, 1965. [in Russian]

2. Akhiezer N.I. Lectures on integral transforms. Kharkov: Vishcha Shkola, 1984. [in Russian]

3. Arestov V.V. On integral inequalities for trigonometric polynomials and their derivatives // Math. USSR-Izv., 1982. Vol. 18, no. 1. P. 1-17. DOI: 10.1070/IM1982v018n01ABEH001375

4. Arestov V.V. Sharp inequalities for trigonometric polynomials with respect to integral functionals // Proc. Steklov Inst. Math., 2011. Vol. 273, suppl. 1. P. 21-36. DOI: 10.1134/S0081543811050038

5. Arestov V.V., Glazyrina P.Yu. Bernstein-Szegö inequality for fractional derivatives of trigonometric polynomials // Proc. Steklov Inst. Math., 2015. Vol. 288, suppl. 1. P. 13-28. DOI: 10.1134/S0081543815020030

6. Boas R.P., Jr. Entire functions. New York: Academic Press, 1954.

7. Bisgaard T.M., Sasvári Z. Characteristic functions and moment sequences: positive definiteness in probability. New York: Nova Sci. Publishers, 2000. 
8. Gashkov S.B. Bernstein's inequality, Riesz's identity and Euler's formula for the sum of reciprocal squares // Mat. Pros., Ser. 3, 2014. Vol. 18. P. 143-171.

9. Gorin E.A. Bernstein inequalities from the operator theory point of view // Vestnik Kharkov. Univ. Prikl. Mat. Mekh.,1980. Vol. 45. P. 77-105.

10. Kahane J.-P. Séries de Fourier absolument convergentes. Berlin: Springer, 1970.

11. Kozko A.I. The exact constants in the Bernstein-Zygmund-Szegö inequalities with fractional derivatives and the Jackson-Nikolskii inequality for trigonometric polynomials // East J. Approx., 1998. Vol. 4, no. 3. P. 391-416.

12. Lieb E., Loss M. Analysis. Graduete Studies in Math., vol. 14. AMS, Providence, Rhode Island, 1997.

13. Lizorkin P.I. Bounds for trigonometrical integrals and an inequality of Bernstein for fractional derivatives // Izv. Akad. Nauk SSSR Ser. Mat., 1965. Vol. 29, no. 1. P. 109-126.

14. Lukacs E. Characteristic functions. London: Griffin, 1970.

15. Manov A., Zastavnyi V. Positive definiteness of piecewise-linear function // Expositiones Mathematicae, 2017. Vol. 35. P. 357-361. DOI: 10.1016/j.exmath.2016.12.002

16. Riesz M. Formule d'interpolation pour la dérivée d'un polynome trigonométrique // C. R. Acad. Sci. 1914. Vol. 158. P. 1152-1154.

17. Riesz M. Eine trigonometrische interpolationsformel und einige ungleichungen für polynome // Jahresbericht der Deutschen Mathematiker-Vereinigung, 1914. Vol. 23. P. 354-368.

18. Rudin W. Real and complex analysis. Singapore: McGraw-Hill Int. Editions, 3nd Ed., 1987.

19. Sasvári Z. Positive definite and definitizable functions. Berlin: Akademie Verlag, 1994.

20. Szegö G. Über einen Satz des Herrn Serge Bernstein // Schriften der Königsberger Gelehrten Gesellschaft. 1928. Vol. 5, no. 4. P. 59-70.

21. Sz.-Nagy B. Über gewisse Extremalfragen bei transformierten trigonometrischen Entwicklungen. I. Periodischer Fall // Berichte der Sächsischen Akademie der Wissenschaften zu Leipzig, 1938. Vol. 90. P. 103-134.

22. Timan A.F. Theory of approximation of functions of a real variable. Oxford: Pergamon Press, 1963.

23. Trigub R.M., Belinsky E.S. Fourier analysis and approximation of functions. Dordrecht: Kluwer Acad. Publ., 2004.

24. Vakhaniya N.N., Tarieladze V.I., and Chobanyan S.A. Probability distributions in Banach spaces. M.: Nauka, 1985. [in Russian]

25. Vinogradov O.L. Sharp error estimates for the numerical differentiation formulas on the classes of entire functions of exponential type // Siberian Math. J., 2007. Vol. 48, no. 3. P. 430-445. DOI: $10.1007 / \mathrm{s} 11202-007-0046-9$

26. Williamson R.E. Multiply monotone functions and their Laplace transforms // Duke Math. J., 1956. Vol. 23, no. 2. P. 189-207. DOI: 10.1215/S0012-7094-56-02317-1

27. Zastavnyi V.P. On positive definiteness of some functions // Journal of Multivariate Analysis, 2000. Vol. 73, no. 1. P. 55-81. DOI: 10.1006/jmva.1999.1864

28. Zygmund A. Trigonometric series. Vol. II. Cambridge: Cambridge Univ. Press, 1959. 